\title{
INVARIANT MEASURES FOR CONTACT HAMILTONIAN SYSTEMS: SYMPLECTIC SANDWICHES WITH CONTACT BREAD
}

\author{
A. BRAVETTI, M. DE LEÓN, J. C. MARRERO, AND E. PADRÓN
}

\begin{abstract}
We prove that, under some natural conditions, Hamiltonian systems on a contact manifold $C$ can be split into a Reeb dynamics on an open subset of $C$ and a Liouville dynamics on a submanifold of $C$ of codimension 1 . For the Reeb dynamics we find an invariant measure. Moreover, we show that, under certain completeness conditions, the existence of an invariant measure for the Liouville dynamics can be characterized using the notion of a symplectic sandwich with contact bread.
\end{abstract}

\section{INTRODUCTION}

Contact Hamiltonian systems have been the subject of intense study over the last years. This is in part because contact structures have found applications in many areas of science, ranging e.g. from classical and quantum mechanics [7, 13, 14, 17, 22 to field theories [15, 21, from statistical mechanics 11 to statistics [3, and from optimization [8] to celestial mechanics, cosmology and relativity [1, 9, 31, 39, 40.

One question that is of particular interest in the study of such systems is the existence of invariant measures for the flow. This is undoubtedly one of the most important questions about dynamical systems in general. In fact, for a dynamical system with $n$ degrees of freedom, an invariant measure and $(n-2)$ first integrals which are independent on a level set, we have that the solutions of the system in the level set may be found by quadratures (see, for instance, 30] and the references therein). So, for this reason, invariant measures for interesting special dynamical systems have been intensively discussed in literature (see e.g. 34 for Poisson Hamiltonian systems, and 20] and the references therein for non-holonomic mechanical systems).

Recently, some methods have been proposed for the integration of contact Hamiltonian systems (see for example 25). Invariant measures for contact Hamiltonian systems are a good tool in order to solve this problem. Moreover, the existence of these measures is also interesting because a positive answer could be used, in conjunction with the geometric discretization methods proposed in [9, 37, 42, to develop new algorithms for statistical physics (e.g. Nosé-Hoover-like methods for molecular dynamics [11) and for statistics (e.g. for using contact Hamiltonian dynamics in the context of Hamiltonian Monte Carlo methods [2, 3), and it could be also relevant for the question of the existence of attractors in inflationary cosmology [39, 40].

Contrary to standard symplectic Hamiltonian systems, for which Liouville's theorem states the existence of a natural invariant volume, the situation for contact flows is more involved, as we shall see shortly. A partial answer to the existence of an invariant measure for contact flows has been given in [10, where the authors have found an analogue of Liouville's theorem for contact flows (cf. Theorem 2 in [10]). However, such theorem provides an invariant measure that is well-defined only in the region where the Hamiltonian is different from zero, whereas the corresponding statement in 10] concerning the (invariant) region where the Hamiltonian is identically zero (cf. Theorem 1 therein) is incorrect. Therefore it remains open the question of exploring

Key words and phrases. Contact manifold, contact Hamiltonian system, Reeb dynamics, exact symplectic manifold, Liouville vector field, invariant measure.

2020 Mathematics Subject Classification. 37A05; 37J55; 53D10; 70G45; $70 \mathrm{H} 05$. 
conditions for the existence of an invariant measure in the region where the contact Hamiltonian vanishes identically.

Motivated by these arguments, in this work we start an analysis of the existence of invariant measures for contact flows. To do this, one may proceed directly or one may consider the symplectification of the contact structure and, then, discuss the problem in the setting of homogeneous symplectic manifolds. In fact, contact structures and, more generally, non-coorientable contact structures may be considered as homogeneous symplectic structures. Here, the homogeneity is associated with a principal $\mathbb{R}^{\times}$-bundle structure (a nice and detailed discussion of this fact may be found in [12]). Anyway, in this paper, we opt for the first option and we deal with the problem directly in the contact manifold.

In fact, we proceed in two steps:

- In the first step, we provide a suitable description of the contact Hamiltonian dynamics. In particular, we prove that, in the open subset $U$, where the Hamiltonian function $H$ has no zeros, the Hamiltonian vector field $X_{H}$ is the Reeb vector field of a conformal change of the original contact form $\eta$ (Theorem 3.1).

On the other hand, if the Reeb vector field $\xi$ associated with $\eta$ is transverse to the zero level set $S$ of $H$, then we see that the differential of the restriction of $\eta$ to $S$ induces an exact symplectic structure on $S$ and the restriction of $X_{H}$ to $S$ is, up to reparametrization, the Liouville vector field of the exact symplectic manifold $S$ (Theorem 3.3).

- In the second step, our strategy in order to discuss the existence of an invariant measure for $X_{H}$ will be to study it separately on the regions $U$ and $S$. Since $X_{H \mid U}$ is the Reeb vector field of a conformal change of the contact structure $\eta$, we directly find an invariant measure for $X_{H \mid U}$ and we recover the corresponding result in [10] (see Corollary 4.2).

On the other hand, the restriction of $X_{H}$ to $S$ does not admit, in general, an invariant measure. For instance, if $X_{H \mid S}$ has a critical point then there cannot be any invariant measure for $X_{H \mid S}$ (Corollary 4.5). However under the assumptions that $\xi(H)=\gamma \in$ $\mathbb{R}-\{0\}$ and that both $\xi$ and $X_{H \mid S}$ are complete, we prove in Theorem 4.14, as the main result of this paper, that $X_{H \mid S}$ admits an invariant measure if and only if the original contact manifold $C$ is a symplectic sandwich with contact bread (see Definition 4.12) and $X_{H \mid S}$ admits a suitable global rectification in the sandwich.

The previous results are related to the theory of convex hypersurfaces of contact manifolds (see [19, 23]) and the theory of Weinstein and convex contact structures (see [16, 19, 36, 45]). In fact, explicit relations are presented in Remarks 3.5 and 4.11 and in the last section of the paper (Section 5). Our analysis is also similar to the "Sasaki-Kähler sandwich" appearing in Sasakian geometry [5] and has some common points with [46, 47], where the author discusses the case of manifolds equipped with closed degenerate 2 -forms admitting contact points.

The paper is organized as follows. In Section 2 we review some results and constructions on Hamiltonian dynamics in contact manifolds which will be used in the rest of the paper. In Section 3 , we will prove that, under natural conditions, the Hamiltonian dynamics on a contact manifold $C$ is a Reeb dynamics in the open subset $U$ of $C$ where the Hamiltonian function is different from zero, plus a Liouville dynamics on the invariant submanifold $S$ in which the Hamiltonian is identically zero. In Section 4, we will discuss the existence of invariant measures for the Reeb and Liouville dynamics in $U$ and $S$, respectively. We will assume that the Reeb vector filed $\xi$ of $C$ and the restriction $X_{H \mid S}$ to $S$ of the Hamiltonian vector field $X_{H}$ are complete. The paper ends with an appendix where we will present the analogous results for the more general case when $\xi$ and $X_{H \mid S}$ are not necessarily complete.

Acknowledgements: Lemma 4.8 and its proof were proposed to one of the authors (JCM) by Jesús Alvarez-López. This result is one of the key points in order to obtain the relation between the symplectic sandwich with contact bread and the existence of an invariant measure for the restriction of the Hamiltonian vector field to the zero level set of the Hamiltonian function. The authors are 
very grateful for the invaluable comments of Jesús Alvarez-López. The authors are also grateful to Kevin Sackel for his comments on the relationship between our results and the theory of convex contact structures and to Charles Boyer and Lin Wang for their valuable comments. JCM and EP are supported by Ministerio de Ciencia e Innovación (Spain) under grants PGC2018-098265-B-C32. MdL has been supported by Ministerio de Ciencia e Innovación (Spain) under grants MTM201676702-P and "Severo Ochoa Programme for Centres of Excellence" in R\&D (SEV-2015-0554). This research was partially supported by the University of La Laguna, that sponsored the visit of AB.

\section{HAMILTONIAN DYNAMICS ON CONTACT MANIFOLDS}

In this section, we will review some results and constructions on Hamiltonian dynamics in contact manifolds (for more details, see for instance [4, 7, 18, 24, 32, 41]).

Let $\eta$ be a 1 -form on a $(2 n+1)$-dimensional manifold $C$. Then $\eta$ is a contact form if the $(2 n+1)$-form

$$
\eta \wedge(d \eta)^{n}
$$

defines a volume form on $C$. The couple $(C, \eta)$ is said to be $\boldsymbol{a}$ contact manifold. In such a case, we can consider the Reeb vector field $\xi \in \mathfrak{X}(C)$ associated with $(C, \eta)$ which is characterized by the conditions

$$
\eta(\xi)=1 \text { and } \iota_{\xi} d \eta=0 .
$$

An alternative characterization of a contact structure is the following: for a 1-form $\eta$ on $C$, we can consider the vector bundle morphism $b_{\eta}: T C \rightarrow T^{*} C$ from the tangent bundle to the cotangent bundle of $C$ given by

$$
b_{\eta}\left(v_{x}\right)=\iota_{v_{x}}((d \eta)(x))+\eta(x)\left(v_{x}\right) \eta(x), \quad \text { for } v_{x} \in T_{x} C \text { with } x \in C .
$$

Then, $\eta$ is a contact 1 -form on $C$ if and only if $b_{\eta}$ is a vector bundle isomorphism. Note that if $(C, \eta)$ is a contact manifold then, using (2.2) and (2.3), we deduce that $b_{\eta}(\xi)=\eta$. Moreover, associated with the contact 1-form $\eta$, we have the 2-vector $\Lambda_{\eta}$ on $C$ given by

$$
\Lambda_{\eta}(\alpha, \beta)=d \eta\left(b_{\eta}^{-1}(\alpha), b_{\eta}^{-1}(\beta)\right) \text { with } \alpha, \beta \in \Omega^{1}(C) .
$$

On the other hand, it is well-known [24 that for every point $x$ on a contact manifold $(C, \eta)$, there are Darboux coordinates $\left(z, q^{1}, \ldots, q^{n}, p_{1}, \ldots, p_{n}\right)$ on an open neighborhood of $x$ such that the local expression of the contact 1 -form $\eta$ is

$$
\eta=d z-\sum_{i=1}^{n} p_{i} d q^{i}
$$

Therefore, the local expressions of the Reeb vector field $\xi$ and the 2-vector $\Lambda_{\eta}$ in such coordinates are

$$
\xi=\frac{\partial}{\partial z} \quad \text { and } \quad \Lambda_{\eta}=\sum_{i=1}^{n}\left(\frac{\partial}{\partial q^{i}}+p_{i} \frac{\partial}{\partial z}\right) \wedge \frac{\partial}{\partial p_{i}} .
$$

Example 2.1 (Contactification versus symplectification). Suppose that $(M, d \lambda)$ is an exact symplectic manifold, then $d z+\lambda$ defines a contact form on $\mathbb{R} \times M$. In this case, the Reeb vector is $\frac{\partial}{\partial z}$ and the contact manifold $(\mathbb{R} \times M, d z+\lambda)$ is called the contactification of $(M, d \lambda)$.

On the other hand, if $\eta$ is a 1 -form on $C$ and $c \in \mathbb{R}-\{0\}$, then one may prove that the 2-form $\Omega_{c}=\exp (c s)(d \eta+c d s \wedge \eta)$ is a symplectic structure on $\mathbb{R} \times C$ if and only if $\eta$ is a contact structure on $C$. In the particular case when $c=-1$, the symplectic manifold ( $\mathbb{R} \times C, \Omega_{1}$ ) is said to be the symplectification of the contact manifold $(C, \eta)[28]$.

Using the 2-vector $\Lambda_{\eta}$ and the Reeb vector field $\xi$, we can introduce a bracket $\{\cdot, \cdot\}_{\eta}: C^{\infty}(C) \times$ $C^{\infty}(C) \rightarrow C^{\infty}(C)$ on the space of differentiable functions on $C$ defined as

$$
\{f, g\}_{\eta}=\Lambda_{\eta}(d f, d g)+f \xi(g)-g \xi(f), \quad \forall f, g \in C^{\infty}(C) .
$$


Note that $\{\cdot,-1\}=\xi$. This bracket is not in general a Poisson bracket because it is not a derivation in each argument with respect to the standard product of real functions. In fact, $\left(C^{\infty}(C),\{\cdot, \cdot\}_{\eta}\right)$ is a Lie algebra and, for all $H \in C^{\infty}(C),\{\cdot, H\}_{\eta}$ defines a first order differential operator, i.e.

$$
\left\{f f^{\prime}, H\right\}_{\eta}=f\left\{f^{\prime}, H\right\}_{\eta}+f^{\prime}\{f, H\}_{\eta}-f f^{\prime}\{1, H\}_{\eta}, \quad \forall f, f^{\prime} \in C^{\infty}(C) .
$$

Therefore $\{\cdot, \cdot\}_{\eta}$ is a Jacobi bracket (see [27, 29, 33]). Moreover, the operator $\{\cdot, H\}_{\eta}: C^{\infty}(C) \rightarrow$ $C^{\infty}(C)$ can be identified with a couple $\left(X_{H}, f_{H}\right)$, where $X_{H}$ is a vector field and $f_{H}$ is a function on $C$, which are related with $\{\cdot, H\}_{\eta}$ by the formula

$$
\{f, H\}_{\eta}=X_{H}(f)+f_{H} f .
$$

A direct computation proves that

$$
X_{H}=-\iota_{d H} \Lambda_{\eta}-H \xi \text { and } f_{H}=\xi(H) .
$$

The vector field $X_{H}$ is called the Hamiltonian vector field associated with $H$. In fact, the Hamiltonian vector field $X_{H}$ is the unique vector field on $C$ characterized by

$$
\iota_{X_{H}} d \eta=d H-\xi(H) \eta \quad \text { and } \quad \iota_{X_{H}} \eta=-H .
$$

The integral curves of $X_{H}$ are the solutions of the contact Hamiltonian dynamical system $(C, \eta, H)$. It is clear that the Reeb vector field is the Hamiltonian vector field associated with the constant function -1 .

Using Darboux local coordinates $\left(z, q^{i}, p_{i}\right)$ on $C$, and Einstein's notation implying a sum over repeated indices, the Hamiltonian vector field $X_{H}$ is just

$$
X_{H}=\frac{\partial H}{\partial p_{i}} \frac{\partial}{\partial q^{i}}-\left(\frac{\partial H}{\partial q^{i}}+p_{i} \frac{\partial H}{\partial z}\right) \frac{\partial}{\partial p_{i}}+\left(p_{i} \frac{\partial H}{\partial p_{i}}-H\right) \frac{\partial}{\partial z}
$$

and the contact Hamiltonian equations associated with $X_{H}$, are given by

$$
\frac{d q^{i}}{d t}=\frac{\partial H}{\partial p_{i}}, \quad \frac{d p_{i}}{d t}=-\left(\frac{\partial H}{\partial q^{i}}+p_{i} \frac{\partial H}{\partial z}\right), \quad \frac{d z}{d t}=p_{i} \frac{\partial H}{\partial p_{i}}-H .
$$

The nature of contact Hamiltonian dynamics is different from the nature of symplectic Hamiltonian dynamics. In fact, for a contact Hamiltonian system $(C, \eta, H)$ we have the following results:

- $H$ is not a first integral of $X_{H}$. Indeed, using (2.4), we deduce

$$
X_{H}(H)=-H \xi(H) \text {. }
$$

- From (2.5), it follows that

$$
\mathcal{L}_{X_{H}} \eta=\iota_{X_{H}} d \eta+d\left(\iota_{X_{H}} \eta\right)=-\xi(H) \eta .
$$

Therefore, the contact form $\eta$ is not invariant under the action of $X_{H}$.

- The Liouville volume $\nu=\eta \wedge(d \eta)^{n}$ is not invariant under the action of $X_{H}$. In fact, using (2.7), we have that

$$
\mathcal{L}_{X_{H}} \nu=-\xi(H) \nu+n \eta \wedge d\left(\mathcal{L}_{X} \eta\right) \wedge(d \eta)^{n-1}=-(n+1) \xi(H) \nu .
$$

However, $\eta$ and $\nu$ are invariant under the action of $\xi=X_{-1}$, i.e.

$$
\mathcal{L}_{\xi} \eta=0 \text { and } \mathcal{L}_{\xi} \nu=0 .
$$

Next, we will present some examples of interesting contact Hamiltonian systems.

Example 2.2 (Dissipative mechanical systems). We consider the product manifold $C=$ $\mathbb{R} \times T^{*} \mathbb{R}^{2}$ endowed with the canonical contact structure $\eta=d z-p_{1} d q^{1}-p_{2} d q^{2}$, where $z$ and $(\mathbf{q}, \mathbf{p})=\left(q^{1}, q^{2}, p_{1}, p_{2}\right)$ are global coordinates on $\mathbb{R}$ and $T^{*} \mathbb{R}^{2}$, respectively. The contact Hamiltonian function $H: C \rightarrow \mathbb{R}$ is given by

$$
H(z, \mathbf{q}, \mathbf{p})=\frac{1}{2}\left(p_{1}^{2}+p_{2}^{2}\right)+V(\mathbf{q})+\gamma z, \quad \text { with } \gamma \in \mathbb{R}-\{0\} .
$$


So, we have that the contact Hamiltonian equations are

$$
\frac{d q^{i}}{d t}=p_{i}, \quad \frac{d p_{i}}{d t}=-\frac{\partial V}{\partial q^{i}}-\gamma p_{i}, \quad \text { with } \quad i=1,2 \quad \text { and } \quad \frac{d z}{d t}=\left(p_{1}^{2}+p_{2}^{2}\right)-H
$$

or, equivalently,

$$
\ddot{q}^{i}+\gamma \dot{q}^{i}+\frac{\partial V}{\partial q^{i}}=0 \quad \text { with } \quad i=1,2, \quad \text { and } \quad \frac{d z}{d t}=\frac{1}{2}\left(\left(\dot{q}^{1}\right)^{2}+\left(\dot{q}^{2}\right)^{2}\right)-V(\mathbf{q})-\gamma z
$$

The reader may recognize the typical second order differential equation associated with a dissipative mechanical system, where the dissipation is linear in the velocity (see e.g. 7, 17, 22, for further details).

Example 2.3 (Reeb dynamics and standard Hamiltonian systems). Let $Q$ be a smooth manifold and $\omega_{Q}=-d \lambda_{Q}$ the canonical symplectic structure on $T^{*} Q$, with $\lambda_{Q}$ the Liouville 1form on $T^{*} Q$. Suppose that $H: T^{*} Q \rightarrow \mathbb{R}$ is a Hamiltonian function and $\mathcal{H}_{H} \in \mathfrak{X}\left(T^{*} Q\right)$ is the symplectic Hamiltonian vector field associated with $H$. This means that $\mathcal{H}_{H}$ is defined by the following condition

$$
\iota \mathcal{H}_{H} \omega_{Q}=d H \text {. }
$$

Denote by $\Delta$ the Liouville vector field on $T^{*} Q$, which is defined by

$$
\iota_{\Delta} \omega_{Q}=-\lambda_{Q} \text {. }
$$

Let $c \in \mathbb{R}$ be a real number such that $\Delta(H) \neq 0$ on $H^{-1}(c)$. Then $C=H^{-1}(c)$ is a submanifold of $T^{*} Q$ of codimension 1 (note that the condition $\Delta(H) \neq 0$ on $H^{-1}(c)$ implies that $H$ is regular at every point of $\left.H^{-1}(c)\right)$. Moreover, using that $H$ is a first integral of $\mathcal{H}_{H}$, it follows that the restriction of $\mathcal{H}_{H}$ to $C$ is tangent to $C$. In addition, since $\lambda_{Q}\left(\mathcal{H}_{H}\right)=\Delta(H)$, we have that $\left(\lambda_{Q}\left(\mathcal{H}_{H}\right)\right)_{\mid C} \neq 0$, at every point of $C$. Thus, we may use Theorem 5.9 in [24] and deduce the following results:

- The 1 -form $\eta=i_{C}^{*} \lambda_{Q}$ is a contact form on $C$, where $i_{C}: C \rightarrow T^{*} Q$ is the canonical inclusion.

- The Reeb vector field of the contact manifold $(C, \eta)$ is the vector field $\xi$ on $C$ given by

$$
\xi=\frac{\left(\mathcal{H}_{H}\right)_{\mid C}}{\Delta(H)_{\mid C}}
$$

So, in conclusion, the restriction to $C$ of the symplectic Hamiltonian dynamics $\mathcal{H}_{H}$ is just a re-parametrization of the contact Hamiltonian vector field on $C$ corresponding to the constant function -1 , that is, the Reeb dynamics.

\section{Contact Hamiltonian systems and Reeb-Liouville dynamics}

Let $(C, \eta, H)$ be a contact Hamiltonian system on a manifold $C$ of dimension $2 n+1$. Denote by $U$ the open subset of $C$

$$
U=\{x \in C / H(x) \neq 0\}
$$

and by $i_{U}: U \rightarrow C$ the canonical inclusion. Then, in this section, we will prove the following facts:

- It is possible to define another contact form on $U$ so that the restriction of the Hamiltonian vector field $X_{H}$ to $U$ is just the Reeb vector field of the new contact form.

- Under reasonable hypotheses, one has that the complementary subset $S=C-U$ of $U$ is a submanifold of codimension 1 of $C$ which admits an exact symplectic structure.

- The restriction of $X_{H}$ to $S$ is tangent to $S$ and $X_{H \mid S}$ is a reparametrization of the Liouville vector field of the exact symplectic manifold $S$.

In conclusion, the contact Hamiltonian dynamics is confined to the two complementary regions $U$ and $S$, and it is a Reeb dynamics on $U$ and a Liouville dynamics on $S$.

Next, we will prove the first result. 
Theorem 3.1. The 1-form

$$
\eta_{H}=-\frac{1}{H \circ i_{U}} i_{U}^{*} \eta
$$

defines a contact structure on $U$. Moreover, the Reeb vector field of $\eta_{U}$ is just the restriction $X_{H \mid U}$ of the Hamiltonian vector field associated with $H$ to $U$.

Proof. A direct computation proves that

$$
d \eta_{H}=\frac{1}{\left(H \circ i_{U}\right)^{2}} d\left(H \circ i_{U}\right) \wedge i_{U}^{*} \eta-\frac{1}{H \circ i_{U}} i_{U}^{*} d \eta .
$$

Therefore,

$$
\eta_{H} \wedge\left(d \eta_{H}\right)^{n}=\frac{(-1)^{n+1}}{\left(H \circ i_{U}\right)^{n+1}} i_{U}^{*}\left(\eta \wedge(d \eta)^{n}\right),
$$

is a volume form on $U$ and hence $\eta_{H}$ is a contact 1-form on $U$. Furthermore, from (2.5), it follows that

$$
\eta_{H}\left(X_{H \mid U}\right)=-\frac{1}{H \circ i_{U}}\left(\eta\left(X_{H}\right) \circ i_{U}\right)=1 .
$$

On the other hand, if $\xi$ is the Reeb vector field of $C$ then, using (2.5), (2.6) and (3.1), we deduce that

$\iota_{X_{H \mid U}} d \eta_{H}=-\frac{1}{H \circ i_{U}}\left(\xi(H) \circ i_{U}\right) i_{U}^{*} \eta+\frac{1}{H \circ i_{U}} d\left(H \circ i_{U}\right)-\frac{1}{H \circ i_{U}}\left(d\left(H \circ i_{U}\right)-\left(\xi(H) \circ i_{U}\right) i_{U}^{*} \eta\right)=0$.

This proves the result.

Now, a natural question arises: what happens in the subset $S=C-U=H^{-1}(0)$ ? In order to give an answer to this question, we start with a preliminary result, which is a consequence from (2.6).

Lemma 3.2. If $S \neq \emptyset$ and 0 is a regular value of $H$, then $S$ is a submanifold of $C$ of codimension 1 which is invariant under the action of $X_{H}$, that is, $X_{H \mid S}$ is tangent to $S$.

After Lemma 3.2. two natural questions arise:

(i) What is the induced geometric structure on $S$ ?

(ii) What is the description of $X_{H \mid S}$ in terms of the previous structure?

Related to these two questions, we may prove the following result.

Theorem 3.3. Let $H: C \rightarrow \mathbb{R}$ be a Hamiltonian function on a contact manifold $(C, \eta)$ with Reeb vector field $\xi$ and assume that $S=H^{-1}(0) \neq \emptyset$ and that $\xi(H)(x) \neq 0$, for all $x \in S$.

(i) If $\theta=i_{S}^{*} \eta$ on $S$, with $i_{S}: S \rightarrow C$ the inclusion map, then $\Omega=d \theta$ is an exact symplectic structure on $S$.

(ii) If $\Delta$ is the Liouville vector field of the exact symplectic manifold $(S, \Omega)$, i.e. $\Delta$ is the vector field characterized by

$$
\iota_{\Delta} \Omega=\theta
$$

then $X_{H \mid S}$ is the reparametrization of $\Delta$ given by

$$
X_{H \mid S}=-\left(\xi(H) \circ i_{S}\right) \Delta .
$$

Proof. The condition $\xi(H)(x) \neq 0$ for all $x \in S$ implies that 0 is a regular value of $H$ and, using Lemma 3.2, we deduce that $S$ is a submanifold of $C$ of codimension 1 and $X_{H \mid S}$ is a vector field on $S$.

(i) We will see that $\Omega$ is a non-degenerate 2-form on $S$. In fact, if $x \in S$ and $v \in T_{x} S$ satisfies

$$
\iota_{v} \Omega(x)=0
$$


we have that $\left(\iota_{v} d \eta(x)\right)(u)=0$ for all $u \in T_{x} S$. Now, since $\xi$ is transverse to the submanifold $S$, it follows that

$$
T_{x} C=T_{x} S \oplus\langle\xi(x)\rangle .
$$

However, $\iota_{v} d \eta(x)=0$ implies $v \in\langle\xi(x)\rangle$, and hence $v \in\langle\xi(x)\rangle \cap T_{x} S$ which, by (3.5), means that $v=0$.

(ii) Using (2.5) and the fact that $i_{S}^{*}(d H)=0$, we obtain that

$$
\iota_{X_{H \mid S}} \Omega=i_{S}^{*}(d H-\xi(H) \eta)=-\left(\xi(H) \circ i_{S}\right) \theta=\iota_{-\left(\xi(H) \circ i_{S}\right) \Delta} \Omega .
$$

Therefore, since $\Omega$ is a non-degenerate 2 -form, we conclude that $X_{H \mid S}=-\left(\xi(H) \circ i_{S}\right) \Delta$.

Remark 3.4. Note that if $\xi(H) \equiv 0$ then the behavior of $X_{H}$ is similar to that of a Hamiltonian vector field in an exact presymplectic manifold $\left(C^{2 n+1}, d \eta\right)$ of dimension $2 n+1$ (of rank $2 n$ ). In fact, from (2.6), (2.7) and (2.8), we deduce that:

- $H$ is a first integral of $X_{H}$ and

- The contact structure $\eta$ and the Liouville volume $\nu=\eta \wedge(d \eta)^{n}$ are $X_{H}$-invariant.

Remark 3.5. Under the same hypotheses as in Theorem 3.3, we have that:

- $S=H^{-1}(0)$ is a convex hypersurface of the contact manifold $(C, \eta)$ (see [19, 23] for the theory of convex hypersurfaces).

- If, in addition, we assume that $\xi(H)$ is a positive function on $C$, then it is easy to prove that there exists a Riemannian metric $g$ on $C$ such that $\xi(H)=\|\xi\|^{2}$ and $d H$ is the dual metric of $\xi$ with respect to $g$. So, if we take $0<\delta \leq \frac{1}{2}$, it follows that

$$
\xi(H) \geq \delta\left(\|\xi\|^{2}+\|d H\|^{2}\right) .
$$

This implies that $(\xi, H)$ is a gradient-like pair and that $(C, \eta, \xi, H)$ is a convex contact manifold (without singular points) in the terminology by Sackel (see [36]).

\section{INVARIANT MEASURES FOR CONTACT HAMILTONIAN DYNAMICS}

Let $(C, \eta, H)$ be a Hamiltonian system on a connected contact manifold $C$ of dimension $2 n+1$. Then, $C$ is orientable and a (positive) volume form on $C$ is just the Liouville volume $\nu=\eta \wedge(d \eta)^{n}$. On the other hand, any positive volume form on $C$ is given by

$$
\nu_{\sigma}=\exp (\sigma) \nu=\exp (\sigma) \eta \wedge(d \eta)^{n}
$$

with $\sigma: C \rightarrow \mathbb{R} \in C^{\infty}(C)$.

Proposition 4.1. The volume form $\nu_{\sigma}$ is an invariat measure for the Hamiltonian vector field $X_{H}$ if and only if

$$
X_{H}(\sigma)=(n+1) \xi(H),
$$

where $\xi$ is the Reeb vector field associated with $(C, \eta)$.

Proof. The volume form $\nu_{\sigma}$ is an invariant measure for $X_{H}$ if and only if $\mathcal{L}_{X_{H}} \nu_{\sigma}=0$.

Now, using (2.8), we deduce that

$$
\mathcal{L}_{X_{H}} \nu_{\sigma}=X_{H}(\sigma) \nu_{\sigma}+\exp (\sigma) \mathcal{L}_{X_{H}} \nu=\left(X_{H}(\sigma)-(n+1) \xi(H)\right) \nu_{\sigma},
$$

which implies the result. 
If $\left(z, q^{i}, p_{i}\right)$ are Darboux coordinates for the contact manifold $(C, \eta)$, we have that the local expression of Equation (4.1) is

$$
\frac{\partial H}{\partial p_{i}} \frac{\partial \sigma}{\partial q^{i}}-\left(\frac{\partial H}{\partial q^{i}}+p_{i} \frac{\partial H}{\partial z}\right) \frac{\partial \sigma}{\partial p_{i}}+\left(p_{i} \frac{\partial H}{\partial p_{i}}-H\right) \frac{\partial \sigma}{\partial z}=(n+1) \frac{\partial H}{\partial z}
$$

for which finding an explicit form for the solutions seems to be rather prohibitive.

So, our strategy in order to discuss the existence of invariant measures for the contact Hamiltonian dynamics will be to study it separately on the regions

$$
U=\{x \in C / H(x) \neq 0\} \text { and } S=C-U .
$$

Since the restriction $X_{H \mid U}$ is just the Reeb vector field of the contact form $\eta_{H}=-\frac{1}{H \circ i_{U}} i_{U}^{*} \eta$ (see Theorem 3.1), then the Liouville volume induced by $\eta_{H}$ is invariant under the action of $X_{H \mid U}$, that is

$$
\mathcal{L}_{X_{H \mid U}}\left(\eta_{H} \wedge\left(d \eta_{H}\right)^{n}\right)=0 .
$$

Therefore, using (3.2), we deduce the following result which was proved in [10].

Corollary 4.2. The volume form $\frac{1}{H^{n+1}} \eta \wedge(d \eta)^{n}$ on $U$ is an invariant measure for $X_{H \mid U}$, which corresponds to the function $\sigma=-(n+1) \ln (H)$ in Proposition 4.1 .

Now, in order to discuss the existence of an invariant measure on $S=H^{-1}(0)=C-U$, we will assume the hypothesis

$$
\xi(H)(x) \neq 0 \quad \text { for all } x \in S .
$$

Then, using Theorem 3.3 , we have that the restriction of $X_{H}$ to the closed submanifold $S$ is tangent to $S$ and

$$
X_{H \mid S}=-\left(\xi(H) \circ i_{S}\right) \Delta
$$

where $i_{S}: S \rightarrow C$ is the inclusion map and $\Delta$ denotes the Liouville vector field of the exact symplectic manifold $\left(S, d i_{S}^{*} \eta\right)$.

Moreover, we may prove that the existence of an invariant measure for the contact Hamiltonian dynamics on $S$ is equivalent to the existence of an invariant measure for the Liouville vector field.

Lemma 4.3. $\Delta$ preserves a volume form $\nu_{S}$ on $S$ if and only if $X_{H \mid S}$ preserves the volume form $\frac{1}{\xi(H) \circ i_{S}} \nu_{S}$.

Proof. Indeed, using the properties of the Lie derivative, we deduce that

$$
\mathcal{L}_{\Delta} \nu_{S}=-\mathcal{L}_{\frac{X_{H \mid S}}{\xi(H) \circ i_{S}}} \nu_{S}=-d\left(\frac{1}{\xi(H) \circ i_{S}}\right) \wedge \iota_{X_{H \mid S}} \nu_{S}-\frac{1}{\xi(H) \circ i_{S}} \mathcal{L}_{X_{H \mid S}} \nu_{S},
$$

and

$$
\mathcal{L}_{X_{H \mid S}}\left(\frac{1}{\xi(H) \circ i_{S}} \nu_{S}\right)=X_{H \mid S}\left(\frac{1}{\left.\xi(H) \circ i_{S}\right)}\right) \nu_{S}+\frac{1}{\xi(H) \circ i_{S}} \mathcal{L}_{X_{H \mid S}} \nu_{S} .
$$

Now, since $d\left(\frac{1}{\xi(H) \circ i_{S}}\right) \wedge \nu_{S}$ is a $(2 n+1)$-form on $S$, we have that $0=d\left(\frac{1}{\xi(H) \circ i_{S}}\right) \wedge \nu_{S}$ and thus

$$
0=\iota_{X_{H \mid S}}\left(d\left(\frac{1}{\xi(H) \circ i_{S}}\right) \wedge \nu_{S}\right)=X_{H \mid S}\left(\frac{1}{\xi(H) \circ i_{S}}\right) \nu_{S}-d\left(\frac{1}{\xi(H) \circ i_{S}}\right) \wedge \iota_{X_{H \mid S}} \nu_{S},
$$

that is,

$$
X_{H \mid S}\left(\frac{1}{\xi(H) \circ i_{S}}\right) \nu_{S}=d\left(\frac{1}{\xi(H) \circ i_{S}}\right) \wedge \iota_{X_{H \mid S}} \nu_{S}
$$


So, using (4.2) and (4.3), we conclude that

$$
\mathcal{L}_{\Delta} \nu_{S}=-\mathcal{L}_{X_{H \mid S}}\left(\frac{1}{\xi(H) \circ i_{S}} \nu_{S}\right)
$$

which proves the result.

Therefore, our problem reduces to discussing the existence of an invariant measure for the Liouville vector field $\Delta$ of the exact symplectic manifold $\left(S, d i_{S}^{*} \eta\right)$. Using this fact, we may deduce the following result.

Theorem 4.4. Let $(C, \eta, H)$ be a contact Hamiltonian system on a contact manifold $(C, \eta)$ of dimension $2 n+1$ with Reeb vector field $\xi$. If $\xi(H)(x) \neq 0$ for every $x \in S=H^{-1}(0)$, then the restriction to $S$ of the contact Hamiltonian dynamics $X_{H}$ admits an invariant measure if and only if there exists a real $C^{\infty}$-function $\sigma$ on $S$ such that

$$
X_{H \mid S}(\sigma)=n\left(\xi(H) \circ i_{S}\right),
$$

where $i_{S}: S \rightarrow C$ is the canonical inclusion. Moreover, if the previous condition holds then

$$
\frac{\exp (\sigma)}{\xi(H) \circ i_{S}}\left(d i_{S}^{*} \eta\right)^{n}
$$

is an invariant measure for $X_{H \mid S}$.

Proof. From the first item in Theorem 3.3 , we have that $\left(S, d i_{S}^{*} \eta\right)$ is an exact symplectic manifold of dimension $2 n$ and a (positive) volume form $\nu_{S}$ on $S$ is given by

$$
\nu_{S}=\exp (\sigma)\left(d i_{S}^{*} \eta\right)^{n}
$$

with $\sigma: S \rightarrow \mathbb{R} \in C^{\infty}(S)$.

Now, if $\Delta$ is the Liouville vector field on $S$ then, using the fact that $\iota_{\Delta} d\left(i_{S}^{*} \eta\right)=i_{S}^{*} \eta$, we deduce that

$$
\mathcal{L}_{\Delta} d\left(i_{S}^{*} \eta\right)=d \mathcal{L}_{\Delta}\left(i_{S}^{*} \eta\right)=d\left(\iota_{\Delta} d\left(i_{S}^{*} \eta\right)+d\left(\left(i_{S}^{*} \eta\right)(\Delta)\right)\right)=d i_{S}^{*} \eta .
$$

Thus, using (4.5) and the properties of the Lie derivative, we obtain that

$$
\mathcal{L}_{\Delta} \nu_{S}=(\Delta(\sigma)+n) \nu_{S}
$$

and, therefore,

$$
\mathcal{L}_{\Delta} \nu_{S}=0 \quad \Leftrightarrow \quad \Delta(\sigma)=-n
$$

On the other hand, from Lemma 4.3, it follows that

$$
\mathcal{L}_{X_{H \mid S}}\left(\frac{1}{\xi(H) \circ i_{S}} \nu_{S}\right)=0 \quad \Leftrightarrow \quad \mathcal{L}_{\Delta} \nu_{S}=0 .
$$

In addition, since $X_{H \mid S}=-\left(\xi(H) \circ i_{S}\right) \Delta$, we deduce that

$$
X_{H \mid S}(\sigma)=n\left(\xi(H) \circ i_{S}\right) \Leftrightarrow \Delta(\sigma)=-n .
$$

Thus, the result follows using (4.5), 4.6), (4.7) and (4.8).

An immediate consequence of the previous theorem is the following obstruction to the existence of a (global) invariant measure on $S$.

Corollary 4.5. Let $(C, \eta, H)$ be a contact Hamiltonian system on a contact manifold $(C, \eta)$ of dimension $2 n+1$ with Reeb vector field $\xi$. If $\xi(H)(x) \neq 0$ for every $x \in S=H^{-1}(0)$ and $X_{H \mid S}$ has a critical point, then there cannot exist any invariant measure for $X_{H \mid S}$.

Proof. If $x_{0} \in S$ is a critical point of $X_{H \mid S}$ and $\sigma$ is an arbitrary real $C^{\infty}$-function on $S$ then $X_{H}(\sigma)\left(x_{0}\right)=0$. This, using Theorem 4.4 and the fact $\xi(H)\left(x_{0}\right) \neq 0$, proves the result. 
After Corollary 4.5, a natural question arises: what happens if $X_{H}(x) \neq 0$ for every point $x \in S$ or, equivalently (see (3.4) ), if $\Delta(x) \neq 0$ for every $x \in S$ ?

The previous question may be reformulated as follows. Suppose that $(S, d \theta)$ is an exact symplectic manifold, that $\Delta$ is the Liouville vector field of $S$ and that $\Delta(x) \neq 0 \forall x \in S$. Then, our question is:

Is there a real $C^{\infty}$-function $\sigma$ on $S$ such that $\Delta(\sigma)=-n$ ?

It is clear that the previous question is equivalent to the following one:

Is there a real $C^{\infty}$-function $\sigma$ on $S$ such that $\Delta(\sigma)=1$ ?

Next, we will consider two particular examples.

Example 4.6 (The cotangent bundle without the zero section). Suppose that $Q$ is a smooth manifold of dimension $n$ and that our symplectic manifold is the open subset $S$ of the cotangent bundle $T^{*} Q$ of $Q$ given by $S=T^{*} Q-0_{Q}(Q)$, where $0_{Q}: Q \rightarrow T^{*} Q$ is the zero section. On $S$, we consider the restriction of the symplectic structure $\omega_{Q}=-d \lambda_{Q}$ on $T^{*} Q$, with $\lambda_{Q}$ the Liouville 1-form. If $\Delta$ is the Liouville vector field on $S$ and $\left(q^{i}, p_{i}\right)$ are fibred coordinates on $S$, we have that

$$
\lambda_{Q}=p_{i} d q^{i}, \omega_{Q}=d q^{i} \wedge d p_{i}, \quad \Delta=p_{i} \frac{\partial}{\partial p_{i}} .
$$

So, it is clear that $\Delta(x) \neq 0$, for every $x \in S$.

Now, we take a Riemannian metric $g$ on $Q$,

$$
g=g_{i j}(q) d q^{i} \otimes d q^{j}, \quad \text { with } g_{i j}=g\left(\frac{\partial}{\partial q^{i}}, \frac{\partial}{\partial q^{j}}\right)
$$

and the kinetic energy $K_{g}: T^{*} Q \rightarrow \mathbb{R}$ associated with $g$ given by

$$
K_{g}(\alpha)=\frac{1}{2}\|\alpha\|^{2}, \quad \text { for } \alpha \in T^{*} Q .
$$

If $\left(g^{i j}(q)\right)$ is the inverse matrix of $\left(g_{i j}(q)\right)$, we have that

$$
K_{g}(q, p)=\frac{1}{2} g^{i j}(q) p_{i} p_{j}
$$

From (4.9) and (4.10), it follows that

$$
\Delta\left(\left(K_{g}\right)_{\mid S}\right)=2\left(K_{g}\right)_{\mid S} .
$$

Thus, if $\sigma: S \rightarrow \mathbb{R}$ is the real function on $S$ defined by $\sigma=\frac{1}{2} \ln \left(K_{g}\right)_{\mid S}+\left(F \circ \pi_{Q}\right)_{\mid S}$, with $F: Q \rightarrow \mathbb{R}$ a function on $Q$ and $\pi_{Q}: T^{*} Q \rightarrow Q$ the canonical projection, we deduce that

$$
\Delta(\sigma)=1 .
$$

Note that, in fact, the volume form $\nu_{S}$ on $S$ given by

$$
\nu_{S}=\exp (-n \sigma) j_{S}^{*}\left(d \lambda_{Q}\right)^{n}=\left(\left(K_{g}\right)_{\mid S}^{-\frac{n}{2}} \exp \left(-n\left(F \circ \pi_{Q}\right){ }_{\mid S}\right) j_{S}^{*}\left(d \lambda_{Q}\right)^{n}\right.
$$

is an invariant measure for $\Delta$, with $j_{S}: S \rightarrow T^{*} Q$ the canonical inclusion. In particular, if $F(q)=-\frac{1}{n} \ln (\rho(q))$, with $\rho(q)$ some distribution on $Q$, we get

$$
\nu_{S}=\left(\left(K_{g}\right)_{\mid S}^{-\frac{n}{2}}\left(\rho \circ \pi_{Q}\right)_{\mid S}\right) j_{S}^{*}\left(d \lambda_{Q}\right)^{n},
$$

which provides an invariant measure of the type needed in Hamiltonian Monte Carlo algorithms [2].

Example 4.7 (Dissipative mechanical systems (revisited)). Consider the framework of Example 2.2. If $\xi$ is the Reeb vector field of the canonical contact structure on $C$, we have that

$$
\xi(H)=\frac{\partial H}{\partial z}=\gamma \neq 0 .
$$


For this system, the closed submanifold $S$ of $C$ is

$$
S=H^{-1}(0)=\left\{\left(-\frac{1}{\gamma}\left(\frac{1}{2}\left(p_{1}^{2}+p_{2}^{2}\right)+V(\mathbf{q})\right), \mathbf{q}, \mathbf{p}\right) \in C /(\mathbf{q}, \mathbf{p}) \in T^{*} \mathbb{R}^{2}\right\} \simeq T^{*} \mathbb{R}^{2},
$$

and the 1-form $\theta$ and the Liouville vector field $\Delta$ are given by

$$
\theta=i_{S}^{*} \eta=-\sum_{i=1}^{2}\left[\left(\frac{1}{\gamma} \frac{\partial V}{\partial q^{i}}+p_{i}\right) d q^{i}+\frac{1}{\gamma} p_{i} d p_{i}\right]
$$

and

$$
X_{H \mid S}=-\gamma \Delta=\sum_{i=1}^{2}\left[p_{i} \frac{\partial}{\partial q^{i}}-\left(\gamma p_{i}+\frac{\partial V}{\partial q^{i}}\right) \frac{\partial}{\partial p_{i}}\right] .
$$

Thus, if $\mathbf{p} \neq 0$ then $X_{H \mid S}(\mathbf{q}, \mathbf{p}) \neq 0$. On the other hand, $X_{H \mid S}(\mathbf{q}, \mathbf{0})=0$ if and only if $\mathbf{q}$ is a critical point of $V$. So, if $V$ has a critical point then there cannot be an invariant measure for $X_{H \mid S}$.

Now, we consider the particular case when $V(\mathbf{q})=q^{1}+q^{2}$. Then, $V$ has no critical points and, using Theorem 4.4, we have that

$$
X_{H \mid S}=\sum_{i=1}^{2}\left[p_{i} \frac{\partial}{\partial q^{i}}-\left(\gamma p_{i}+1\right) \frac{\partial}{\partial p_{i}}\right]
$$

admits an invariant measure if and only if there exists a real $C^{\infty}$-function $\sigma$ on $S$ such that $X_{H \mid S}(\sigma)=2 \gamma$ or, equivalently

$$
p_{1} \frac{\partial \sigma}{\partial q^{1}}+p_{2} \frac{\partial \sigma}{\partial q^{2}}-\left(\gamma p_{1}+1\right) \frac{\partial \sigma}{\partial p_{1}}-\left(\gamma p_{2}+1\right) \frac{\partial \sigma}{\partial p_{2}}=2 \gamma
$$

A solution of this equation is

$$
\sigma(\mathbf{q}, \mathbf{p})=-\gamma\left(p_{1}+p_{2}\right)-\gamma^{2}\left(q^{1}+q^{2}\right)
$$

Thus (see Theorem 4.4),

$$
\frac{1}{\gamma} \exp \left(-\gamma\left(p_{1}+p_{2}\right)-\gamma^{2}\left(q^{1}+q^{2}\right)\right) d q^{1} \wedge d p_{1} \wedge d q^{2} \wedge d p_{2}
$$

is an invariant measure for $X_{H \mid S}$.

Next we will show that, in a situation like the previous example (that is, $\xi(H)=\gamma \neq 0$ ), the existence of a function $\sigma: S \rightarrow \mathbb{R}$ such that $X_{H \mid S}(\sigma)=n \gamma$ is equivalent to a certain trivialization $\mathbb{R} \times B$ of $S$ under which $X_{H \mid S}$ is a reparametrization of $\frac{\partial}{\partial s}$, where $s$ is the global coordinate on $\mathbb{R}$.

In the proof of such a result, we will use the following fact.

Lemma 4.8. Let $Z$ be a complete vector field on a manifold $M$. Then, the following sentences are equivalent:

(i) There is a function $\sigma \in C^{\infty}(M)$ such that $Z(\sigma)=r$, with $r \in \mathbb{R}-\{0\}$.

(ii) There exist a submanifold $D$ of $M$ of codimension 1 and a diffeomorphism $\varphi: M \rightarrow \mathbb{R} \times D$ such that, if $t$ is the global coordinate on $\mathbb{R}$,

$$
T_{y} \varphi\left(Z_{y}\right)=\left.\frac{\partial}{\partial t}\right|_{\varphi(y)}
$$

for all $y \in D$.

In fact, if (i) holds then $D=\sigma^{-1}(0)$ and the inverse map to $\varphi$ is just the restriction to $\mathbb{R} \times D$ of the flow $\Phi^{Z}$ of the vector field $Z$. 
Proof. Suppose that there is a function $\sigma \in C^{\infty}(M)$ such that $Z(\sigma)=r \neq 0$. Then 0 is a regular value of $\sigma$ and $D=\sigma^{-1}(0)$ is a submanifold of $M$ of codimension 1. Now, we consider the smooth map

$$
\varphi: M \rightarrow \mathbb{R} \times D, \quad \varphi(y)=\left(\frac{1}{r} \sigma(y), \Phi^{Z}\left(-\frac{1}{r} \sigma(y), y\right)\right), \quad y \in M,
$$

where $\Phi^{Z}: \mathbb{R} \times M \rightarrow M$ is the flow of $Z$.

Note that, since $Z(\sigma)=r$ then, by integration, we deduce that

$$
\sigma\left(\Phi_{t}^{Z}(y)\right)=r t+\sigma(y), \quad \text { for all } y \in M
$$

As a consequence, $\sigma\left(\Phi^{Z}\left(-\frac{1}{r} \sigma(y), y\right)\right)=-\sigma(y)+\sigma(y)=0$, that is, $\Phi^{Z}\left(-\frac{1}{r} \sigma(y), y\right) \in D$.

On the other hand, if $(t, x) \in \mathbb{R} \times D$ then, using (4.14) and the fact that $\sigma(x)=0$, we have that

$$
\begin{aligned}
\varphi\left(\Phi^{Z}(t, x)\right) & =\left(\frac{1}{r} \sigma\left(\Phi^{Z}(t, x)\right), \Phi^{Z}\left(-\frac{1}{r} \sigma\left(\Phi^{Z}(t, x)\right), \Phi^{Z}(t, x)\right)\right) \\
& =\left(t+\frac{1}{r} \sigma(x), \Phi^{Z}\left(-t-\frac{1}{r} \sigma(x), \Phi^{Z}(t, x)\right)\right)=(t, x) .
\end{aligned}
$$

Moreover, if $y \in M$,

$$
\Phi^{Z}(\varphi(y))=\Phi^{Z}\left(\frac{1}{r} \sigma(y), \Phi^{Z}\left(-\frac{1}{r} \sigma(y), y\right)\right)=\Phi^{Z}(0, y)=y .
$$

Therefore, $\varphi$ is bijective and $\varphi^{-1}$ is just the restriction of $\Phi^{Z}$ to $\mathbb{R} \times D$. Finally, using this last fact and that $\left(T_{(t, x)} \Phi^{Z}\right)\left(\frac{\partial}{\partial t} \mid(t, x)\right)=Z_{\mid \Phi^{Z}(t, x)}$ for $(t, x) \in \mathbb{R} \times D$, we conclude that

$$
T_{y} \varphi\left(Z_{y}\right)=\frac{\partial}{\partial t}_{\mid \varphi(y)} .
$$

Conversely, if there exists a diffeomorphism $\varphi: M \rightarrow D \times \mathbb{R}$ with $D$ a submanifold of $M$ of codimension 1 such that (4.13) holds, then the function $\sigma: M \rightarrow \mathbb{R}$ is just $\sigma=p_{r} \circ \varphi, p_{r}: \mathbb{R} \times D \rightarrow \mathbb{R}$ being the map $p_{r}(t, x)=r t$. The relation $Z(\sigma)=r$ is deduced from (4.13).

Now, we apply the previous lemma to a particular class of contact Hamiltonian systems.

Proposition 4.9. Let $(C, \eta)$ be a contact manifold with complete Reeb vector field $\xi$ and $H: C \rightarrow \mathbb{R}$ a Hamiltonian function such that $\xi(H)(x)=\gamma \neq 0$ for all $x \in C$. Then, there exists a contact isomorphism $\varphi_{1}: C \rightarrow \mathbb{R} \times S$ from the contact manifold $(C, \eta)$ to the contactification $\left(\mathbb{R} \times S, d z+i_{S}^{*} \eta\right)$ of the exact symplectic manifold $\left(S, d_{S}^{*} \eta\right)$, i.e. $\varphi_{1}$ is a diffeomorphism and

$$
\varphi_{1}^{*}\left(d z+i_{S}^{*} \eta\right)=\eta
$$

where $i_{S}: S \rightarrow C$ is the inclusion map. Therefore,

$$
T_{y} \varphi_{1}\left(\xi_{y}\right)=\left.\frac{\partial}{\partial z}\right|_{\varphi_{1}(y)}, \quad \text { for all } y \in C .
$$

Proof. In order to obtain the diffeomorphism $\varphi_{1}: C \rightarrow \mathbb{R} \times S$, we apply Lemma 4.8 to the manifold $C$ and the vector field $\xi$. Then, there is a diffeomorphism $\varphi_{1}: C \rightarrow \mathbb{R} \times S$ such that

$$
T_{y} \varphi_{1}\left(\xi_{y}\right)=\left.\frac{\partial}{\partial z}\right|_{\mid \varphi_{1}(y)}
$$

Moreover, if $\Phi_{\mid \mathbb{R} \times S}^{\xi}: \mathbb{R} \times S \rightarrow C$ is the restriction of the flow of $\xi$ to $\mathbb{R} \times S$, then $\varphi_{1}$ is just $\left(\Phi_{\mid \mathbb{R} \times S}^{\xi}\right)^{-1}$ and

$$
\left(\Phi_{\mid \mathbb{R} \times S}^{\xi}\right)^{*}(\eta)\left(\frac{\partial}{\partial z}\right)=\eta(\xi)=1
$$


In addition, using that $\mathcal{L}_{\xi} \eta=0$, we deduce that

$$
\mathcal{L}_{\frac{\partial}{\partial z}}\left[\left(\Phi_{\mid \mathbb{R} \times S}^{\xi}\right)^{*}(\eta)\right]=0
$$

Therefore, from 4.15) and 4.16), we obtain

$$
\left(\Phi_{\mid \mathbb{R} \times S}^{\xi}\right)^{*}(\eta)=d z+\alpha
$$

with $\alpha$ a 1 -form on $S$.

Now, we consider the map $i_{0}: S \rightarrow \mathbb{R} \times S$, given by $i_{0}(x)=(0, x)$. Then, it is clear that the following diagram

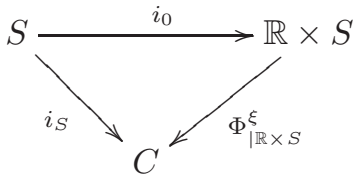

is commutative. Thus, from (4.17), we deduce that

$$
i_{S}^{*} \eta=i_{0}^{*}\left(\left(\Phi_{\mid \mathbb{R} \times S}^{\xi}\right)^{*}(\eta)\right)=i_{0}^{*}(d z)+i_{0}^{*}(\alpha)=\alpha .
$$

As a consequence,

$$
\left(\Phi_{\mid \mathbb{R} \times S}^{\xi}\right)^{*}(\eta)=d z+i_{S}^{*} \eta
$$

Since $\varphi_{1}=\left(\Phi_{\mid \mathbb{R} \times S}^{\xi}\right)^{-1}$, we conclude that

$$
\varphi_{1}^{*}\left(d z+i_{S}^{*} \eta\right)=\eta
$$

If, in addition, we suppose that the reparametrization $\frac{X_{H \mid S}}{\xi(H) \circ i_{S}}$ of $\left(X_{H}\right)_{\mid S}$ is complete, we also have the following result

Proposition 4.10. Let $(C, \eta)$ be a contact manifold of dimension $2 n+1$ and $H: C \rightarrow \mathbb{R} a$ Hamiltonian function such that

(i) The Reeb vector field $\xi$ is complete.

(ii) $\xi(H)(x) \neq 0$ for all $x \in S=H^{-1}(0)$

(iii) The reparametrization $Z=\frac{X_{H \mid S}}{\xi(H) \circ i_{S}}$ of the restriction of the Hamiltonian vector field $X_{H}$ to the submanifold $S$ of $C$ is complete, where $i_{S}: S \rightarrow C$ is the inclusion map.

Then, the following sentences are equivalent:

(a) There is a function $\sigma: S \rightarrow \mathbb{R}$ such that $X_{H \mid S}(\sigma)=n\left(\xi(H) \circ i_{S}\right)$.

(b) There exist a submanifold $B$ of $S$ of codimension 1 with canonical inclusion $i_{B}: B \rightarrow C$ such that $\eta_{B}=i_{B}^{*} \eta$ is a contact structure on $B$ and a symplectomorphism

$$
\varphi_{2}: S \rightarrow \mathbb{R} \times B
$$

from the symplectic manifold $\left(S, d i_{S}^{*} \eta\right)$ to the symplectification of $\left(B, \eta_{B}\right)$, i.e.

$$
\varphi_{2}^{*}\left(\exp (-s)\left(d \eta_{B}-d s \wedge \eta_{B}\right)\right)=d i_{S}^{*} \eta
$$

where $s$ is the global coordinate on $\mathbb{R}$. Moreover,

$$
T_{y} \varphi_{2}\left(\left(\frac{X_{H \mid S}}{\xi(H) \circ i_{S}}\right)_{y}\right)=\frac{\partial}{\partial s}{\mid \varphi_{2}(y)},
$$

for all $y \in S$. 
Proof. If (b) holds, then we can consider the function $\sigma: S \rightarrow \mathbb{R}$ given by

$$
\sigma=n\left(p r_{1} \circ \varphi_{2}\right)
$$

where $p r_{1}: \mathbb{R} \times B \rightarrow \mathbb{R}$ is the canonical projection on the first factor. Thus, using (4.18), it follows that

$$
Z(\sigma)=\frac{X_{H \mid S}}{\xi(H) \circ i_{S}}(\sigma)=n
$$

Next, assume that (a) holds. Then we may apply Lemma 4.8, with $M=S, Z=\frac{X_{H \mid S}}{\xi(H) \circ i_{S}} \in \mathfrak{X}(S)$ and $r=n \in \mathbb{R}-\{0\}$ and we deduce that there exist a submanifold $B=\sigma^{-1}(0)$ of codimension 1 of $S$ and a diffeomorphim $\varphi_{2}: S \rightarrow \mathbb{R} \times B$ such that

$$
T_{y} \varphi_{2}\left(Z_{y}\right)=\left.\frac{\partial}{\partial s}\right|_{\mid \varphi_{2}(y)}, \quad \text { for } y \in S
$$

Moreover, from Theorem 3.3. we conclude that $d i_{S}^{*} \eta$ is an exact symplectic structure on $S$ and

$$
X_{H \mid S}=-\left(\xi(H) \circ i_{S}\right) \Delta
$$

where $\Delta$ is the Liouville vector field of the exact symplectic manifold $\left(S, d i_{S}^{*} \eta\right)$. This implies that $Z=\frac{X_{H \mid S}}{\xi(H) \circ i_{S}}=-\Delta$ and that

$$
\mathcal{L}_{Z}\left(i_{S}^{*} \eta\right)=-i_{S}^{*} \eta
$$

Now, if $i_{B}: B \rightarrow C$ is the inclusion map, we will prove that

$$
\varphi_{2}^{*}\left(\exp (-s)\left(d i_{B}^{*} \eta-d s \wedge i_{B}^{*} \eta\right)\right)=d i_{S}^{*} \eta .
$$

In fact, using (4.19) and (4.21) and the fact that $\Phi_{\mid \mathbb{R} \times B}^{Z}: \mathbb{R} \times B \rightarrow S$ is the inverse map of $\varphi_{2}$, it follows that

or equivalently,

$$
\mathcal{L}_{\frac{\partial}{\partial s}}\left(\Phi_{\mid \mathbb{R} \times B}^{Z}\right)^{*}\left(i_{S}^{*} \eta\right)=-\left(\Phi_{\mid \mathbb{R} \times B}^{Z}\right)^{*}\left(i_{S}^{*} \eta\right),
$$

$$
\mathcal{L}_{\frac{\partial}{\partial s}}\left(\exp (s)\left(\Phi_{\mid \mathbb{R} \times B}^{Z}\right)^{*}\left(i_{S}^{*} \eta\right)\right)=0 .
$$

This implies that

$$
\exp (s)\left(\Phi_{\mid \mathbb{R} \times B}^{Z}\right)^{*}\left(i_{S}^{*} \eta\right)=f d s+\alpha,
$$

where $f \in C^{\infty}(B)$ and $\alpha \in \Omega^{1}(B)$. Then, we consider the map $i_{0}: B \rightarrow \mathbb{R} \times B$, given by $i_{0}(x)=(0, x)$, and the following diagram

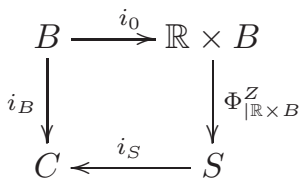

is commutative. Thus, using (4.23), we have that

$$
i_{B}^{*} \eta=\left(i_{S} \circ \Phi_{\mid \mathbb{R} \times B}^{Z} \circ i_{0}\right)^{*} \eta=i_{0}^{*}\left(\left(\Phi_{\mid \mathbb{R} \times B}^{Z}\right)^{*}\left(i_{S}^{*} \eta\right)\right)=i_{0}^{*}(\exp (-s)(f d s+\alpha))=\alpha .
$$

On the other hand, again from (4.23),

$$
d\left(\Phi_{\mid \mathbb{R} \times B}^{Z}\right)^{*}\left(i_{S}^{*} \eta\right)=\exp (-s)(d f \wedge d s+d \alpha)-\exp (-s)(d s \wedge \alpha) .
$$

Therefore,

$$
\iota_{\frac{\partial}{\partial s}} d\left(\Phi_{\mid \mathbb{R} \times B}^{Z}\right)^{*}\left(i_{S}^{*} \eta\right)=-\exp (-s) d f-\exp (-s) \alpha .
$$

Moreover, from (4.19), (4.21) and since $\varphi_{2}$ is the inverse map to $\Phi_{\mid \mathbb{R} \times B}^{Z}$, we have that

$$
\begin{aligned}
\iota_{\frac{\partial}{\partial s}} d\left(\Phi_{\mid \mathbb{R} \times B}^{Z}\right)^{*}\left(i_{S}^{*} \eta\right) & =\iota_{\frac{\partial}{\partial s}}\left(\Phi_{\mid \mathbb{R} \times B}^{Z}\right)^{*}\left(d i_{S}^{*} \eta\right)=\left(\Phi_{\mid \mathbb{R} \times B}^{X_{H \mid S}}\right)^{*}\left(\iota_{Z} d i_{S}^{*} \eta\right) \\
& =-\left(\Phi_{\mid \mathbb{R} \times B}^{Z}\right)^{*}\left(i_{S}^{*} \eta\right)-\left(\Phi_{\mid \mathbb{R} \times B}^{Z}\right)^{*}\left(d \iota_{Z} i_{S}^{*} \eta\right) \\
& =-\left(\Phi_{\mid \mathbb{R} \times B}^{Z}\right)^{*}\left(i_{S}^{*} \eta\right),
\end{aligned}
$$


where the last equation follows using that

$$
\iota_{Z}\left(i_{S}^{*} \eta\right)=-\left(\iota_{\Delta}\left(i_{S}^{*} \eta\right)\right)=0 .
$$

So, from (4.24), (4.26) and (4.27), we deduce that

$$
i_{B}^{*} \eta=i_{0}^{*}\left(\Phi_{\mid \mathbb{R} \times B}^{Z}\right)^{*}\left(i_{S}^{*} \eta\right)=i_{0}^{*}(\exp (-s) d f+\exp (-s) \alpha)=d f+i_{B}^{*} \eta
$$

that is,

$$
d f=0
$$

Substituting (4.24) and (4.28) in (4.25), we have that

$$
\left(\Phi_{\mid \mathbb{R} \times B}^{Z}\right)^{*}\left(d i_{S}^{*} \eta\right)=\exp (-s)\left(d i_{B}^{*} \eta-d s \wedge i_{B}^{*} \eta\right)
$$

which implies (4.22). Thus, since $\left(\Phi_{\mid \mathbb{R} \times B}^{Z}\right)^{*}\left(d i_{S}^{*} \eta\right)$ is a symplectic structure on $\mathbb{R} \times B$, we have that $\eta_{B}=i_{B}^{*} \eta$ is a contact structure on $B$ (see Example 2.1).

Remark 4.11. Under the same hypotheses (i), (ii) and (iii) as in Proposition 4.10 let $\Delta$ be the Liouville vector field of the exact symplectic manifold $\left(S, d\left(i_{S}^{*} \eta\right)\right)$. Using (iii) in such a proposition and (3.4), we have that $\Delta$ is complete. Now, suppose that (a) in Proposition 4.10 holds. This, using again (3.4), implies that $\Delta(\sigma)=-n$. So, proceeding as in Remark 3.5, we deduce that $(\Delta,-\sigma)$ is a gradient-like pair. In addition, $\left(S, d\left(i_{S}^{*} \eta\right), \Delta,-\sigma\right)$ is a Weinstein manifold without singular points (see [16, 19, 36, 45] for the theory of Weinstein manifolds).

Propositions 4.9 and 4.10 motivate the following definition

Definition 4.12. A symplectic sandwich with contact bread consists of

(i) A contact manifold $(C, \eta)$ of dimension $2 n+1$ and two submanifolds $S$ and $B$ of codimension 1 and 2 , respectively, such that the 2 -form $d\left(i_{S}^{*} \eta\right)$ is a symplectic structure on $S$ and the 1-form $i_{B}^{*} \eta$ is a contact structure on $B$, where $i_{S}: S \rightarrow C$ and $i_{B}: B \rightarrow C$ are the canonical inclusions.

(ii) A contact isomorphism

$$
\varphi_{1}: C \rightarrow \mathbb{R} \times S
$$

from the contact manifold $(C, \eta)$ to the contactification $\left(\mathbb{R} \times S, d z+i_{S}^{*} \eta\right)$ of the exact symplectic manifold $\left(S, d i_{S}^{*} \eta\right)$. Here, $z$ is the standard coordinate on $\mathbb{R}$ in $\mathbb{R} \times S$.

(iii) A symplectic isomorphism

$$
\varphi_{2}: S \rightarrow \mathbb{R} \times B
$$

from the symplectic manifold $\left(S, d i_{S}^{*} \eta\right)$ to the symplectification $\left(\mathbb{R} \times B, \exp (-s)\left(d i_{B}^{*} \eta-\right.\right.$ $\left.\left.d s \wedge i_{B}^{*} \eta\right)\right)$ of the contact manifold $\left(B, i_{B}^{*} \eta\right)$. Here, $s$ is the standard coordinate on $\mathbb{R}$ in $\mathbb{R} \times B$.

Remark 4.13. (i) The map

$$
\left(\operatorname{Id}_{\mathbb{R}} \times \varphi_{2}\right): C \rightarrow \mathbb{R} \times(\mathbb{R} \times B)
$$

is a contact isomorphism from the original contact manifold $(C, \eta)$ to the contactification of the symplectification of the contact manifold $\left(B, i_{B}^{*} \eta\right)$. Note that the contact structure on $\mathbb{R} \times(\mathbb{R} \times B)$ is $d z+\exp (-s) i_{B}^{*} \eta$.

(ii) We have the chain of embeddings (the inclusions $i_{S}$ and $\left.\widetilde{i}_{B}\right)$ and projections $\left(p r_{2} \circ \varphi_{1}\right.$ and $\left.p r_{2} \circ \varphi_{2}\right)$

$$
\left.(C, \eta) \stackrel{i_{p r_{2} \circ \varphi_{1}}^{\stackrel{i_{S}}{\rightleftarrows}}\left(S, d\left(i_{S}^{*} \eta\right)\right) \underset{i_{S}}{\stackrel{p r_{2} \circ \varphi_{2}}{\rightleftarrows}}\left(B, i_{B}\right.}{\rightleftarrows} \eta\right)
$$

such that the manifold in the middle of the chain is a symplectic manifold and the manifolds on the left and the one on the right hand of the chain are contact. This is a good 
motivation for using the terminology a symplectic sandwich with contact bread.

For the symplectic sandwich with contact bread we will use the following notation

$$
(C, \eta) \stackrel{\varphi_{1}}{\longrightarrow}\left(\mathbb{R} \times S, d z+i_{S}^{*} \eta\right) \stackrel{\operatorname{Id}_{\mathbb{R}} \times \varphi_{2}}{\longrightarrow}\left(\mathbb{R} \times \mathbb{R} \times B, d z+\exp (-s) i_{B}^{*} \eta\right)
$$

Now, from Propositions 4.9 and 4.10 and Theorem4.4, we deduce the main result of this section.

Theorem 4.14. Let $(C, \eta, H)$ be a contact Hamiltonian system of dimension $2 n+1$ with complete Reeb vector field $\xi$. Suppose that $\xi(H)(x)=\gamma \neq 0$ for all $x \in C$, and that the restriction $X_{H \mid S}$ to $S$ of the contact Hamiltonian dynamics $X_{H}$ is complete. If $i_{S}: S \rightarrow C$ is the canonical inclusion and $\sigma: S \rightarrow \mathbb{R}$ is a $C^{\infty}$-function on $S$, then $X_{H \mid S}$ admits an invariant measure

$$
\nu=\frac{\exp (\sigma)}{\gamma}\left(d i_{S}^{*} \eta\right)^{n}
$$

if and only if we have a symplectic sandwich with contact bread

$$
(C, \eta) \stackrel{\varphi_{1}}{\longrightarrow}\left(\mathbb{R} \times S, d z+i_{S}^{*} \eta\right) \stackrel{I d_{\mathbb{R}} \times \varphi_{2}}{\longrightarrow}\left(\mathbb{R} \times \mathbb{R} \times B, d z+\exp (-s) i_{B}^{*} \eta\right)
$$

and

for all $y \in S$.

$$
T_{y} \varphi_{2}\left(\left(X_{H \mid S}\right)_{y}\right)=\gamma \frac{\partial}{\partial s \mid \varphi_{2}(y)},
$$

Example 4.15 (Dissipative mechanical systems (re-revisited)). In the particular case of Example 4.7, in which $V(\mathbf{q})=q^{1}+q^{2}$, the symplectic sandwich with contact bread is given by

$$
\left(\mathbb{R} \times T^{*} \mathbb{R}^{2}, d z-p_{i} d q^{i}\right) \stackrel{\varphi_{1}}{\longrightarrow} \mathbb{R} \times\left(T^{*} \mathbb{R}^{2}, d q^{i} \wedge d p_{i}\right)
$$

where

$$
\varphi_{1}(z, \mathbf{q}, \mathbf{p})=\left(\frac{1}{2 \gamma}\left(p_{1}^{2}+p_{2}^{2}\right)+\frac{1}{\gamma}\left(q^{1}+q^{2}\right)+z, q^{1}, q^{2}, p_{1}, p_{2}\right) .
$$

Since $\sigma$ is given by (4.12), the submanifold $B$ is just

$$
B=\left\{\left(-q^{2}-\frac{1}{\gamma}\left(p_{1}+p_{2}\right), q^{2}, p_{1}, p_{2}\right) \in T^{*} \mathbb{R}^{2}\right\} \cong \mathbb{R}^{3}
$$

and its contact structure is

$$
\eta_{B}=\left(p_{1}-p_{2}\right) d q^{2}+\frac{1}{\gamma^{2}} d p_{1}+\left(\frac{p_{1}-p_{2}}{\gamma}+\frac{1}{\gamma^{2}}\right) d p_{2}
$$

From (4.11), we have that the flow $\Phi^{X_{H \mid S}}: \mathbb{R} \times T^{*} Q \rightarrow T^{*} Q$ of $X_{H \mid S}$ is just

$$
\Phi^{X_{H \mid S}}(s,(\mathbf{q}, \mathbf{p}))=\left(\frac{1}{\gamma}\left(\mathbf{p}+\frac{1}{\gamma}\right)(1-\exp (-\gamma s))-\frac{1}{\gamma} s+\mathbf{q},\left(\mathbf{p}+\frac{1}{\gamma}\right) \exp (-\gamma s)-\frac{1}{\gamma}\right)
$$

In this case, a straightforward computation proves that the symplectomorphism

$$
\left(T^{*} \mathbb{R}^{2}, d q^{i} \wedge d p_{i}\right) \longrightarrow\left(\mathbb{\varphi _ { 2 }} \longrightarrow B, \exp (-s)\left(d \eta_{B}-d s \wedge \eta_{B}\right)\right)
$$

is given by

$$
\begin{aligned}
\varphi_{2}(\mathbf{q}, \mathbf{p})= & \left(\gamma F(\mathbf{p}, \mathbf{q}), \Phi^{X_{H \mid S}}(-F(\mathbf{p}, \mathbf{q}), \mathbf{q}, \mathbf{p})\right) \\
= & \left(\gamma F(\mathbf{p}, \mathbf{q}), \frac{1}{2 \gamma^{2}}\left[\left(-2 \gamma p_{2}-2\right) \exp (\gamma F)+2+\gamma^{2}\left(q^{2}-q^{1}\right)+\gamma\left(p_{2}-p_{1}\right)\right],\right. \\
& \left.\frac{1}{\gamma}\left[\exp (\gamma F)\left(\gamma p_{1}+1\right)-1\right], \frac{1}{\gamma}\left[\exp (\gamma F)\left(\gamma p_{2}+1\right)-1\right]\right),
\end{aligned}
$$


where $F=\frac{\sigma(\mathbf{q}, \mathbf{p})}{2 \gamma}=-\frac{1}{2}\left(p_{1}+p_{2}+\gamma\left(q^{1}+q^{2}\right)\right)$.

\section{Conclusions And FUture WORK}

In this work we proved various results concerning contact Hamiltonian systems and the existence of invariant measures for such dynamics. In particular, we proved that in the open subset $U$ where the Hamiltonian function $H$ has no zeros, the Hamiltonian vector field $X_{H}$ is the Reeb vector field of a conformal change of the original contact form $\eta$ (Theorem 3.1). This directly implies the existence of an invariant measure for $X_{H \mid U}$, recovering the corresponding result in [10. (see Corollary 4.2). On the other hand, assuming that the Reeb vector field $\xi$ associated with $\eta$ is transverse to the zero level set $S$ of $H$, we showed that $S$ is endowed with an exact symplectic structure and that $X_{H \mid S}$ is, up to reparametrization, the Liouville vector field of the exact symplectic manifold $S$ (Theorem 3.3). Then we used this result to characterize the general condition for the existence of an invariant measure for $X_{H \mid S}$ (see Theorem 4.4). Finally, under the assumptions that $\xi(H)=\gamma \in \mathbb{R}-\{0\}$ and that both $\xi$ and $X_{H \mid S}$ are complete, we proved in Theorem 4.14, as the main result of this paper, that $X_{H \mid S}$ admits an invariant measure if and only if the original contact manifold $C$ is a symplectic sandwich with contact bread (see Definition 4.12) and $X_{H \mid S}$ admits a suitable global rectification in the sandwich.

Our contributions are closely related to the theory of Weinstein and convex contact manifolds without singular points (see Remarks 3.5] and 4.11). Therefore, taking into account that a lot of relevant results have been obtained for the previous manifolds in the presence of singular points (see [16, 36]), it would be interesting to use such results to describe the dynamical behavior of the vector field $X_{H \mid S}$ in the presence of a Weinstein structure on the exact symplectic manifold $S$. We will try to describe the dynamics in the region where there are no singular points and in a neighborhood of each singular point. On the other hand, as another future work, we expect to generalize some of the results to the case where $\xi(H) \neq 0$ is not necessarily a constant (or, more generally, to the case when $(\xi, H)$ is a gradient-like pair following the ideas in [36]) and to understand the relationship with invariant measures stemming from the Lagrangian description of these systems [18, 21, 22, 41, 43, 44. Moreover, given the relevance of contact Hamiltonian systems in many areas of science, we believe that our work will have interesting consequences in various contexts, especially in statistical physics and thermodynamics [6, 11, 26, 35, 38, statistics [3, and inflationary cosmology [39, 40].

\section{Appendix A.}

In Lemma 4.8, we have found the necessary and sufficient conditions for the existence of a function $\sigma \in C^{\infty}(M)$ such that $Z(\sigma)=r \neq 0$, when the vector field $Z$ is complete. In this appendix we will show what happens when we do not assume this completeness condition.

Proposition A.1. Let $Z$ be a vector field on a manifold $M$ and $U$ the maximal open subset of $\mathbb{R} \times M$ such that the flow of $Z$ is defined on $U$. Then, the following sentences are equivalent:

(i) There is a function $\sigma \in C^{\infty}(M)$ such that the graph of the function $-\frac{\sigma}{r}$ is included into $U$ and $Z(\sigma)=r$.

(ii) There exist a submanifold $D$ of $M$ of codimension 1 , an open set $V$ of $\mathbb{R} \times D$ and a diffeomorphism

such that,

$$
\varphi: M \rightarrow V \subset \mathbb{R} \times D
$$

(a) $\left(-p r_{1}(\varphi(y)), y\right) \in U$ for all $y \in M$, where $p r_{1}: \mathbb{R} \times D \rightarrow \mathbb{R}$ is the canonical projection on the first factor.

(b) $V$ is a submanifold of $U$ of codimension 1.

(c) $T_{y} \varphi\left(Z_{y}\right)=\frac{\partial}{\partial t}_{\mid \varphi(y)}$ for all $y \in M$, with $t$ the standard global coordinate on $\mathbb{R}$. 
Proof. As in the proof of Lemma 4.8 if we suppose (i), then we deduce that $D=\sigma^{-1}(0)$ is the submanifold of $M$ which we are looking for. Now, we consider the submanifold of $U$ of codimension 1

$$
V=\{(t, y) \in U / \sigma(y)=0\}=\left(\sigma \circ p r_{2}\right)^{-1}(0)
$$

where $\mathrm{pr}_{2}: U \subseteq \mathbb{R} \times M \rightarrow M$ is the restriction to $U$ of the canonical projection $\mathbb{R} \times M \rightarrow M$ on the second factor. Since $V=U \cap(\mathbb{R} \times D)$, then $V$ is an open subset of $\mathbb{R} \times D$. The diffeomorphism $\varphi$ is constructed as in Lemma 4.8

$$
\varphi: M \rightarrow V \subseteq \mathbb{R} \times D, \quad \varphi(y)=\left(\frac{1}{r} \sigma(y), \Phi^{Z}\left(-\frac{1}{r} \sigma(y), y\right)\right),
$$

where $\Phi^{Z}: U \subseteq \mathbb{R} \times M \rightarrow M$ is the flow of $Z$. Note that, using that the graph of $-\frac{\sigma}{r}$ is contained in $U$ we have that $\left(-\frac{\sigma(y)}{r}, y\right) \in U$ for all $y \in U$. This implies that

$$
\left(\frac{1}{r} \sigma(y), \Phi^{Z}\left(-\frac{1}{r} \sigma(y), y\right)\right) \in U .
$$

Moreover, as in the proof of Lemma 4.8.

$$
\sigma\left(\Phi^{Z}\left(-\frac{1}{r} \sigma(y), y\right)\right)=0
$$

(that is, $\varphi(y) \in \mathbb{R} \times D$ ) and the restriction of $\Phi^{Z}$ to $V$ is the inverse map of $\varphi$.

Conversely, suppose that $D$ is a submanifold of $M$ of codimension $1, V$ is an open subset of $\mathbb{R} \times D$ and $\varphi: M \rightarrow V \subseteq \mathbb{R} \times D$ is a diffeomorphim such that (a), (b) and (c) hold. Then, we consider the function $\sigma=p_{r} \circ \varphi$, where $p_{r}: \mathbb{R} \times D \rightarrow \mathbb{R}$ is the function $p_{r}(t, x)=r t$. From (a) we have that $\operatorname{graph}\left(-\frac{1}{r} \sigma\right) \subseteq U$ and, using (b) and (c), we conclude that $Z(\sigma)=r$.

Using this result we deduce the corresponding versions of Propositions 4.9 and 4.10, when the completeness condition does not hold.

Proposition A.2. Let $(C, \eta, H)$ be a contact Hamiltonian system and $U_{1}$ the maximal open subset of $\mathbb{R} \times C$ such that the flow of the Reeb vector field $\xi$ is defined on $U_{1}$. We suppose that $\xi(H)(x)=$ $\gamma \neq 0$ for all $x \in C$ and that the graph of the function $-\frac{H}{\gamma}$ is contained in $U_{1}$. Then, there exist an open set $V_{1}$ of $\mathbb{R} \times S$ and a contact isomorphism $\varphi_{1}: C \rightarrow V_{1} \subseteq \mathbb{R} \times S$ from the contact manifold $(C, \eta)$ to the contact manifold $\left(V_{1}, i_{V_{1}}^{*}\left(d z+i_{S}^{*} \eta\right)\right)$, i.e.

$$
\varphi_{1}^{*}\left(i_{V_{1}}^{*}\left(d z+i_{S}^{*} \eta\right)\right)=\eta
$$

where $i_{S}: S \rightarrow C$ and $i_{V_{1}}: V_{1} \rightarrow \mathbb{R} \times S$ are the corresponding inclusion maps. Therefore,

$$
T_{y} \varphi_{1}\left(\xi_{y}\right)=\frac{\partial}{\partial z \mid \varphi_{1}(y)}, \text { for all } y \in C .
$$

Proposition A.3. Let $(C, \eta, H)$ be a contact Hamiltonian system of dimension $2 n+1$ and $U_{1}$ the maximal open subset of $\mathbb{R} \times C$ such that the flow of the Reeb vector field $\xi$ is defined on $U_{1}$. Suppose that $\xi(H)(x) \neq 0$ for all $x \in S=H^{-1}(0)$. Then, the following sentences are equivalent:

(a) There is a function $\sigma: S \rightarrow \mathbb{R}$ on $S$ such that $X_{H \mid S}(\sigma)=n\left(\xi(H) \circ i_{S}\right)$ and

$$
\operatorname{graph}\left(-\frac{\sigma}{n}\right) \subseteq U_{2}
$$

with $U_{2}$ the maximal open subset of $\mathbb{R} \times S$ such that the flow of $\frac{X_{H \mid S}}{\xi(H) \circ i_{S}}$ is defined on $U_{2}$. 
(b) There exist a submanifold $B$ of $S$ of codimension 1 , with canonical inclusion $i_{B}: B \rightarrow C$, such that $\eta_{B}=i_{B}^{*} \eta$ is a contact structure on $B$, an open subset $V_{2}$ of $\mathbb{R} \times B$ and a symplectic isomorphism

$$
\varphi_{2}: S \rightarrow V_{2} \subseteq \mathbb{R} \times B
$$

from the symplectic manifold $\left(S, d i_{S}^{*} \eta\right)$ to $\left(V_{2}, i_{V_{2}}^{*}\left(\exp (-s)\left(d \eta_{B}-d s \wedge \eta_{B}\right)\right)\right)$, i.e.

$$
\varphi_{2}^{*}\left(i_{V_{2}}^{*}\left(\exp (-s)\left(d \eta_{B}-d s \wedge \eta_{B}\right)\right)\right)=d\left(i_{S}^{*} \eta\right)
$$

where $s$ is the global coordinate of $\mathbb{R}$ and $i_{V_{2}}: V_{2} \rightarrow \mathbb{R} \times B$ is the inclusion map. Moreover, $\left(\operatorname{pr}_{1}\left(\varphi_{2}(y)\right), y\right) \in U_{2}$ and

$$
T_{y} \varphi_{2}\left(\left(\frac{X_{H \mid S}}{\xi(H) \circ i_{S}}\right)_{y}\right)=\frac{\partial}{\partial s}{\mid \varphi_{2}(y)},
$$

for all $y \in S$.

\section{REFERENCES}

[1] Bautista, Alfredo; Ibort, Alberto; Lafuente, Javier, The contact structure in the space of light rays. A mathematical tribute to Professor José María Montesinos Amilibia, 133-159, Dep. Geom. Topol. Fac. Cien. Mat. UCM, Madrid, (2016).

[2] Betancourt, M., A conceptual introduction to Hamiltonian Monte Carlo. arXiv preprint arXiv:1701.02434 (2017).

[3] Betancourt, M. J., Adiabatic Monte Carlo. arXiv preprint arXiv:1405.3489. (2014).

[4] Boyer, C. P., Completely Integrable Contact Hamiltonian Systems and Toric Contact Structures on $S^{2} \times S^{3}$. SIGMA. Symmetry, Integrability and Geometry: Methods and Applications, 7, 058. (2011).

[5] Boyer, C.; Galicki, K., Sasakian geometry. Oxford Univ. Press. (2008).

[6] Bravetti, A., Contact geometry and thermodynamics. International Journal of Geometric Methods in Modern Physics, 16(supp01), 1940003. (2019).

[7] Bravetti, A.; Cruz, H.; Tapias, D., Contact Hamiltonian mechanics. Annals of Physics, 376, 17-39. (2017).

[8] Bravetti, A.; Daza-Torres, M. L.; Flores-Arguedas, H.; Betancourt, M., Optimization algorithms inspired by the geometry of dissipative systems arXiv preprint arXiv:1912.02928 (2019).

[9] Bravetti, A.; Seri, M.; Vermeeren, M.; Zadra, F., Numerical integration in celestial mechanics: a case for contact geometry. Celestial Mechanics and Dynamical Astronomy, 132(1), 1-29. (2020).

[10] Bravetti, A.; TApias, D., Liouville's theorem and the canonical measure for nonconservative systems from contact geometry. Journal of Physics A: Mathematical and Theoretical, 48 (2015), no. 24, 245001, 11 pp.

[11] Bravetti, A.; TApias, D., Thermostat algorithm for generating target ensembles. Physical Review E, 93(2), 022139. (2016).

[12] Bruce, Andrew James; Grabowska, Katarzyna; Grabowski, Janusz, Remarks on contact and Jacobi geometry. SIGMA Symmetry Integrability Geom. Methods Appl. 13 (2017), Paper No. 059, 22 pp.

[13] Ciaglia, F. M.; Cruz, H.; Marmo, G., Contact manifolds and dissipation, classical and quantum. Ann. Physics 398 (2018), 159-179.

[14] Ciaglia, Florio M.; Di Cosmo, Fabio; Ibort, Alberto; Marmo, Giuseppe; Schiavone, Luca, Covariant variational evolution and Jacobi brackets : particles. Preprint arXiv:2005.04872 to appear in Modern Physics Letters A.

[15] Ciaglia, Florio M.; Di Cosmo, Fabio; Ibort, Alberto; Marmo, Giuseppe; Schiavone, Luca, Covariant variational evolution and Jacobi brackets : fields. Preprint arxiv.org/abs/2005.14429, to appear in Modern Physics Letters A.

[16] Cieliebak, Kai; Eliashberg, Yakov, From Stein to Weinstein and back. Symplectic geometry of affine complex manifolds. American Mathematical Society Colloquium Publications, 59. American Mathematical Society, Providence, RI, 2012..

[17] De León, M.; SARdón, C., Cosymplectic and contact structures for time-dependent and dissipative Hamiltonian systems. Journal of Physics A: Mathematical and Theoretical, 50(25), 255205. (2017).

[18] DE LEÓN, M.; VAlCÁzAR, M. L., Infinitesimal symmetries in contact Hamiltonian systems. Journal of Geometry and Physics, 153, 103651. (2020).

[19] Eliashberg, Yakov; Gromov, Mikhael, Convex symplectic manifolds. Several complex variables and complex geometry, Part 2 (Santa Cruz, CA, 1989), 135-162, Proc. Sympos. Pure Math., 52, Part 2, Amer. Math. Soc., Providence, RI, (1991).

[20] Fedorov, Y. N.; García-Naranjo, L. C.; Marrero, J. C., Unimodularity and preservation of volumes in nonholonomic mechanics. J. Nonlinear Sci. 25 (2015), no. 1, 203-246.

[21] Gaset, J.; Gracia, X.; Muñoz-Lecanda, M. C.; Rivas, X.; Román-Roy, N., A contact geometry framework for field theories with dissipation. Annals of Physics, 414, 168092. (2020). 
[22] Gaset, J.; Gracia, X.; Muñoz-Lecanda, M. C.; Rivas, X.; Román-Roy, N., New contributions to the Hamiltonian and Lagrangian contact formalisms for dissipative mechanical systems and their symmetries. International Journal of Geometric Methods in Modern Physics, 17(06), 2050090. (2020).

[23] Giroux, Emmanuel, Convexité en topologie de contact. (French) [Convexity in contact topology] Comment. Math. Helv. 66 (1991), no. 4, 637-677.

[24] Godbillon, C., Géométrie différentielle et mécanique analytique. (French) Hermann, Paris 1969183 pp.

[25] Grillo, S.; Padrón, E., Extended Hamilton-Jacobi Theory, contact manifolds, and integrability by quadratures J. of Math. Phys. 61, 012901(2020).

[26] Grmela, M.; Öttinger, H. C., Dynamics and thermodynamics of complex fluids. I. Development of a general formalism. Physical Review E, 56(6), 6620. (1997).

[27] Guedira, F.; Lichnerowicz, A., Géométrie des algébres de Lie locales de Kirillov. (French) [Geometry of Kirillov's local Lie algebras] J. Math. Pures Appl. (9) 63 (1984), no. 4, 407-484.

[28] IbÁÑez, R.; De León, M.; Marrero, J. C.; De Diego, D. M., Co-isotropic and Legendre-Lagrangian submanifolds and conformal Jacobi morphisms. J. Phys. A 30 (1997), no. 15, 5427-5444.

[29] Kirillov, A. A., Local Lie algebras. (Russian) Uspehi Mat. Nauk 31 (1976), no. 4(190), 57-76.

[30] Kozlov, V. V., Invariant measures of the Euler-Poincaré equations on Lie algebras. Funct. Anal. Appl. 22 (1988), no. 1, 58-59.

[31] Lazo, M. J.; Paiva, J.; Amaral, J. T.; Frederico, G. S., Action principle for action-dependent Lagrangians toward nonconservative gravity: Accelerating universe without dark energy. Physical Review D, 95(10), 101501. (2017).

[32] Libermann, P.; Marle, C.-M., Symplectic geometry and analytical mechanics. Translated from the French by Bertram Eugene Schwarzbach. Mathematics and its Applications, 35. D. Reidel Publishing Co., Dordrecht, 1987.

[33] Lichnerowicz, André, Les variétés de Jacobi et leurs algébres de Lie associées. (French) J. Math. Pures Appl. (9) 57 (1978), no. 4, 453-488.

[34] Marrero, J.C., Hamiltonian mechanical systems on Lie algebroids, unimodularity and preservation of volumes. J. Geom. Mech. 2 (2010), no. 3, 243-263.

[35] Morrison, P. J., A paradigm for joined Hamiltonian and dissipative systems. Physica D: Nonlinear Phenomena, 18(1-3), 410-419. (1986).

[36] SaCKel, Kevin, Getting a handle on contact manifolds. arXiv preprint arXiv:1905.11965 (2019).

[37] Simoes, A. A.; De Diego, D. M.; De León, M.; Valcázar, M. L., On the geometry of discrete contact mechanics. arXiv preprint arXiv:2003.11892 (2020).

[38] Simoes, A. A.; de León, M.; Valcázar, M. L.; De Diego, D. M., Contact geometry for simple thermodynamical systems with friction. arXiv preprint arXiv:2004.01989 (2020).

[39] Sloan, D., Dynamical similarity. Physical Review D, 97(12), 123541. (2018).

[40] SLOAN, D., Scalar fields and the FLRW singularity. Classical and Quantum Gravity, 36(23), 235004. (2019).

[41] Valcázar, M. L.; DE León, M., Contact Hamiltonian systems. Journal of Mathematical Physics, 60(10), 102902. (2019).

[42] Vermeeren, M.; Bravetti, A.; Seri, M., Contact variational integrators. Journal of Physics A: Mathematical and Theoretical, 52(44), 445206. (2019).

[43] WANG, K.; WANG, L.; YAN, J., Implicit variational principle for contact Hamiltonian systems. Nonlinearity, 30(2), 492. (2016).

[44] Wang, K.; Wang, L.; Yan, J., Aubry-Mather theory for contact Hamiltonian systems. Communications in Mathematical Physics, 366(3), 981-1023. (2019).

[45] Weinstein, Alan, Contact surgery and symplectic handlebodies. Hokkaido Math. J. 20, no. 2, 241-251. (1991).

[46] Zot'ev, D. B., Contact degeneracies of closed 2-forms. Sbornik: Mathematics, 198(4), 491. (2007).

[47] Zot'ev, D. B., Kostant Prequantization of Symplectic Manifolds with Contact Singularities. Mathematical Notes, 105(5-6), 846-863. (2019). 
A. Bravetti: Instituto de Investigaciones en Matemáticas Aplicadas y en Sistemas (IImas), UniverSidad Nacional Autónoma de México, A.P. 70-543, 04510 Ciudad de México, México

E-mail address: alessandro.bravetti@iimas.unam.mx

M. de León: Instituto de Ciencias Matemáticas (CSiC-UAM-UC3M-UCM) and Real Academia de CienCias, C/Nicolás Cabrera 13-15, 28049 Madrid, Spain

E-mail address: mdeleon@icmat.es

J. C. Marrero: Ull-CSiC Geometría Diferencial y Mecánica Geométrica, Departamento de Matemáticas, Estadística e iO, Sección de Matemáticas, Universidad de la laguna, la laguna, Tenerife, Canary Islands, Spain

E-mail address: jcmarrer@ull.edu.es

E. Padrón: UlL-CSiC Geometría Diferencial y Mecánica Geométrica, Departamento de Matemáticas, Estadística e I O, Sección de Matemáticas, Facultad de Ciencias, Universidad de la laguna, la Laguna, Tenerife, Canary Islands, Spain

E-mail address: mepadron@ull.edu.es 